Proceedings of the All-Polish Seminar on Mössbauer Spectroscopy, Warsaw, Poland, June 18-21, 2010

\title{
Impurity Effect on Charge and Spin Density in $\alpha$-Fe - Comparison between Cellular Model, $A b$ Initio Calculations and Experiment
}

\author{
A. BŁACHOWSKI ${ }^{a, *}$ AND U.D. WDOWIK ${ }^{b}$ \\ ${ }^{a}$ Mössbauer Spectroscopy Division, Institute of Physics \\ Pedagogical University, Podchorążych 2, PL-30-084 Kraków, Poland \\ ${ }^{b}$ Applied Computer Science Division, Institute of Technology \\ Pedagogical University, Podchorążych 2, PL-30-084 Kraków, Poland

\begin{abstract}
The influence of the impurity substituted on the regular site in the $\mathrm{BCC} \alpha$-Fe on charge and spin density on the adjacent iron nuclei has been studied by the ab initio method within framework of the full-potential linearized augmented plane-wave formalism applying density functional theorem. Results were correlated with the phenomenological cellular atomic model of Miedema and van der Woude and with the Mössbauer spectroscopy experimental data.
\end{abstract}

PACS: 75.50.Bb, 31.15.A-, 76.80.+y

\section{Introduction}

Impurity substituted on the regular iron site within $\mathrm{BCC} \alpha$-Fe has influence on the charge (electron) density and electron spin density (hyperfine field) on the adjacent iron nuclei. One can study these effects by means of the ${ }^{57} \mathrm{Fe}$ Mössbauer spectroscopy. Namely, the average isomer shift (charge density) and hyperfine field varies with the impurity concentration for random distribution of impurities. Additionally, one can see individual effect of impurities to the second and sometimes to the third co-ordination shell. Hence, the impurity has effect on the average isomer shift $\langle S\rangle$, while individual perturbations to the particular impurity could be described as $\Delta S_{n}$ with the index $n$ denoting subsequent co-ordination shells around the resonant atom. Corresponding perturbations of the spin density influence the average hyperfine field $\langle B\rangle$ and lead to the individual impurity effects $\Delta B_{n}$ [1].

Parameters described above could be determined from the Mössbauer spectrum. Usually, one has to collect a series of spectra versus impurity concentration $c$. On the other hand, one can calculate electron density on the iron nucleus for single impurity - the latter located at various co-ordination shells around resonant atom. Similar calculations could be performed for the spin density, i.e., for the hyperfine field.

The phenomenological cellular atomic model (CAM) of alloys proposed by Miedema and van der Woude $[2,3]$ could be used to estimate the average isomer shift $\left\langle S_{M}\right\rangle$ due to impurities, and to estimate contribution to the isomer shift caused by the impurity in the first co-ordination shell $\Delta S_{1}^{(M)}$. The model relies on the electro-chemical potential and electron density for the Fermi gas of noninteracting fermions.

This contribution concentrates on the correlations between experimental and/or calculated ab initio electron

\footnotetext{
* corresponding author; e-mail: sfblacho@cyf-kr.edu.pl
}

and spin densities and similar quantities obtained within CAM model.

\section{Discussion of results}

Mössbauer data are partly taken from literature (Be [4], Al [5], Si [6], P [7], Ti [8], V [9], Cr [10], Mn [11], Co [12], Ni [11], Ge [13], As [14], Sn [15], Sb [14], W [16], $\operatorname{Re}$ [17], $\mathrm{Pt}[18])$ or obtained in our laboratory $(\mathrm{Cu}[19]$, $\mathrm{Zn}$ [19], Ga [20], Nb [21], Mo [22], Ru [23], Rh [24], Pd [25], Os [26], Ir [27], Au [28]). Spectra obtained in our laboratory were processed by specialized program Gmbernz [21]. The latter program belongs to the MOSGRAF suite. Ab initio calculations of impurities in BCCFe were performed within the spin-polarized density functional theory (DFT). The pseudopotential method with the generalized gradient approximation (GGA) parameterized by Perdew-Burke-Ernzerhof method (PBE) as implemented in the VASP code [29] was used to optimize geometry and atomic positions of the 128-atomic super-cell. Atoms were represented by the projectoraugmented wave pseudopotentials (PAWs) provided by VASP. A plane-wave expansion up to $360 \mathrm{eV}$ was applied. The Brillouin zone of each super-cell containing a defect was sampled using the $3 \times 3 \times 3 \mathrm{k}$-point mesh generated by the Monkhorst-Pack scheme. During defect calculations, the lattice vectors of the super-cell were frozen at the GGA optimized value and the atomic positions were relaxed until the forces acting on all atoms of the super-cell were smaller than $0.01 \mathrm{eV} / \AA$. The total energy was converged down to $0.1 \mathrm{meV} /$ super-cell. Hyperfine parameters were calculated using the full-potential all electron plane wave method (FLAPW) as implemented in the WIEN2k code [30]. These calculations were carried out using the same exchange-correlation approximation and $4 \mathrm{k}$-points in the irreducible part of the Brillouin zone for the tetrahedron method of integration. The muffin-tin radii of particular atoms were set to conform criterion of the almost touching spheres. The cutoff energy expressed as the product of the muffin-tin radii 
and the maximum plane wave vector was equal to 7 and the largest reciprocal vector for the charge Fourier transfer amounted to 12 to guarantee the total energy convergence of the order of $0.01 \mathrm{mRy} /$ super-cell.

Phenomenological CAM approach relies on the following empirical expression [2]:

$$
S=A\left(\Phi_{a}-\Phi_{b}\right)+B\left(\frac{n_{a}-n_{b}}{n_{b}}\right)+S_{b} .
$$

The symbol $S$ denotes isomer shift observed for the alloy containing diluted impurities $a$ in the matrix $b$. The isomer shift is observed on the resonant atoms belonging to the matrix $b$, while impurities $a$ substitute matrix atoms at random. Symbols $\Phi_{a}$ and $\Phi_{b}$ denote respective electrochemical potentials of the pure elements forming binary alloy. Corresponding symbols $n_{a}$ and $n_{b}$ stand for densities of the electrons, the latter treated as non-interacting fermions. Electron density has to be expressed in the units of $4.6 \times 10^{22} \mathrm{~cm}^{-3}[2]$. The symbol $S_{b}$ stands for the isomer shift in the pure matrix. Finally, parameters $A$ and $B$ are to be adjusted basing on the experimental data. Electro-chemical potentials and electron densities relevant for the model are listed for various elements in Ref. [3]. Equation (1) was applied to: (a) the variation of the average isomer shift $d\langle S\rangle / d c$ versus impurity concentration $c$, (b) the experimental first shell perturbation of the isomer shift $\Delta S_{1}^{(E)}$ (averaged over impurity concentration) and (c) the ab initio calculated first shell perturbation of the isomer shift $\Delta S_{1}^{(C)}$. Above quantities were used instead of $S$ in the Eq. (1). Details concerned with the determination of the average hyperfine parameters and $\Delta S_{1}^{(E)}$ from the experimental Mössbauer spectra are described in [21]. Obtained values for the adjustable parameters $A$ and $B$ of CAM are listed in Table. These parameters were used to estimate respective parameters $d\left\langle S_{M}\right\rangle / d c$ and $\Delta S_{1}^{(M)}$ following from CAM. The last parameter $\Delta S_{1}^{(M)}$ has been obtained for experimental data (b) and calculated data (c) - see Fig. 1. Parameters $\Delta S_{1}^{(M)}$ calculated by using experimental input data for the Eq. (1) (case (b)) are slightly different while using $a b$ initio calculated data as input of the Eq. (1) — case (c).

TABLE

Parameters (all $\times 10^{2}$ ) of the CAM obtained for (a), (b) and (c) data sets. See text for details.

\begin{tabular}{c|c|c|c}
\hline \hline & $A$ & $B$ & Dispersion \\
\hline (a) & $0.79 \mathrm{~mm} /(\mathrm{s} \mathrm{V}$ at.\%) & $-2.11 \mathrm{~mm} /(\mathrm{s}$ at.\%) & $0.20 \mathrm{~mm} /(\mathrm{s}$ at.\%) \\
\hline & {$[\mathrm{mm} /(\mathrm{s} \mathrm{V})]$} & {$[\mathrm{mm} / \mathrm{s}]$} & {$[\mathrm{mm} / \mathrm{s}]$} \\
\hline (b) & 3.00 & -11.18 & 2.60 \\
(c) & 4.86 & -13.25 & 1.66
\end{tabular}

Figure 1a shows correlation between experiment and CAM model for $d\langle S\rangle / d c$. A correlation between experiment and CAM for the $\Delta S_{1}$ (due to the first shell) is shown in part (b), while the corresponding correlation between $a b$ initio results and CAM for $\Delta S_{1}$ is shown in part (c).
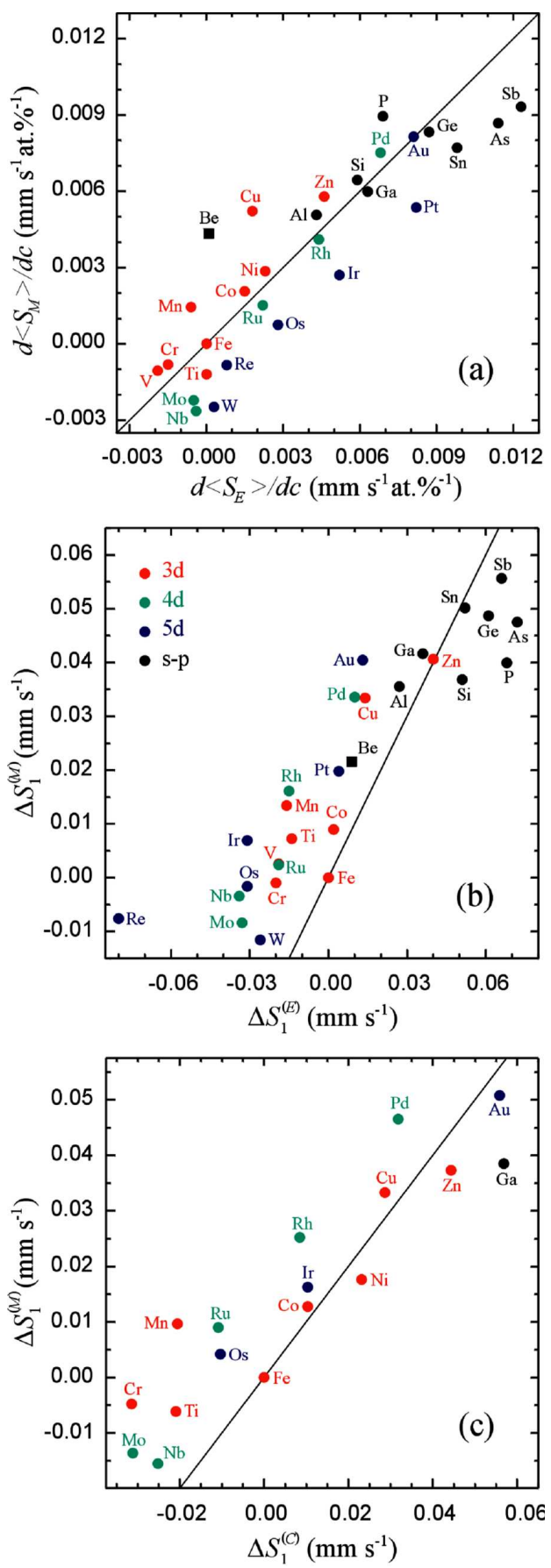

Fig. 1. (a) Correlation between experimental derivative $d\left\langle S_{E}\right\rangle / d c$ of the isomer shift versus impurity concentration $c$ and corresponding derivative within CAM model $d\left\langle S_{M}\right\rangle / d c$. (b) Correlation between experimental first shell perturbations of the isomer shift $\Delta S_{1}^{(E)}$ and $\Delta S_{1}^{(M)}$ obtained basing on the experimental data. (c) Correlation between calculated $\Delta S_{1}^{(C)}$ and $\Delta S_{1}^{(M)}$ obtained basing on the ab initio calculated data. Straight lines show "ideal" correlation. 

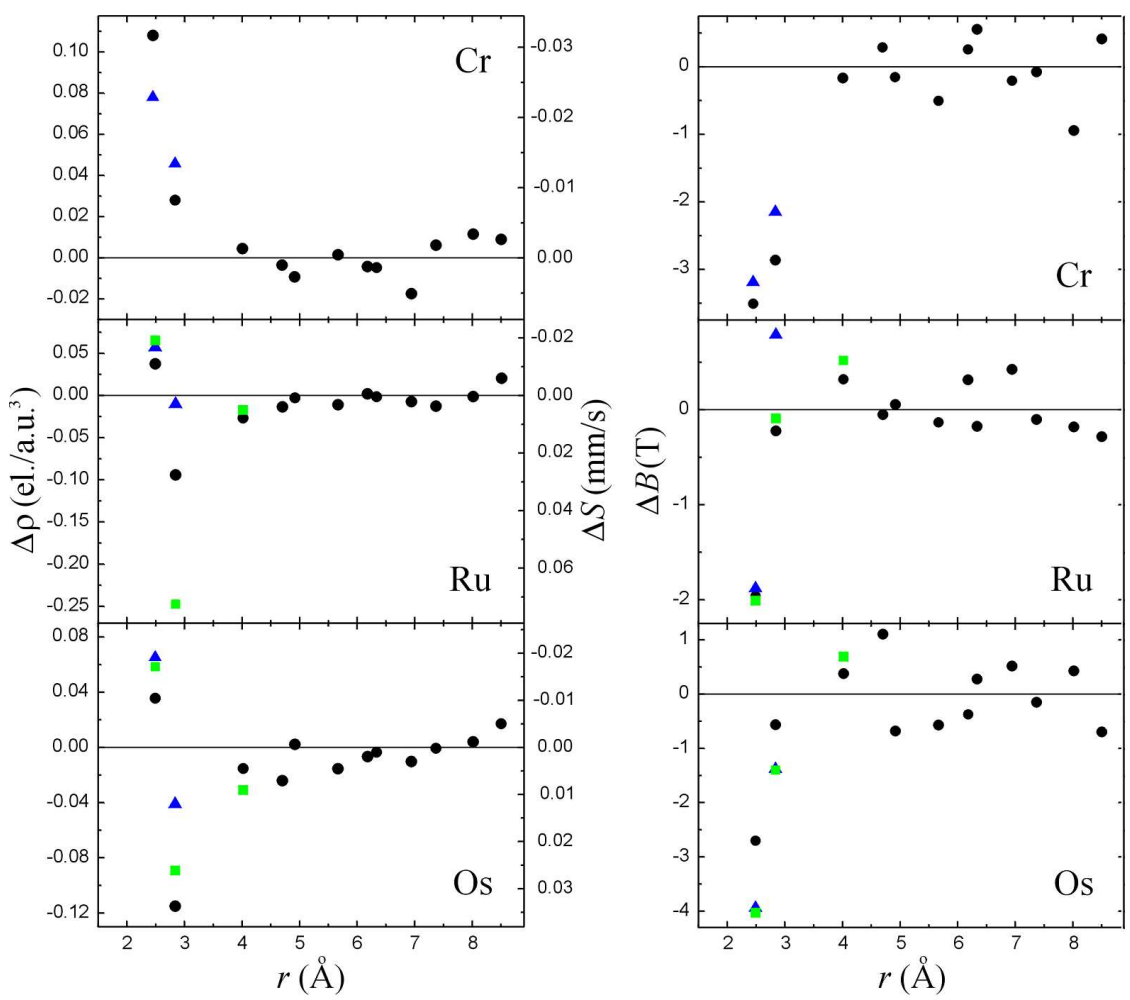

Fig. 2. Variation of the electron density $\Delta \rho$ and corresponding isomer shift $\Delta S$ versus distance $r$ from the impurity (co-ordination shell). Right column shows corresponding variation of the hyperfine field $\Delta B$. Results of the ab initio calculations are shown for $\mathrm{Cr}, \mathrm{Ru}$ and $\mathrm{Os}$. Blue triangles denote experimental data processed to the second co-ordination shell, green rectangles correspond to the experimental data processed to the third shell [10, 23, 26], while black circles are outcome of the $a b$ initio calculations.

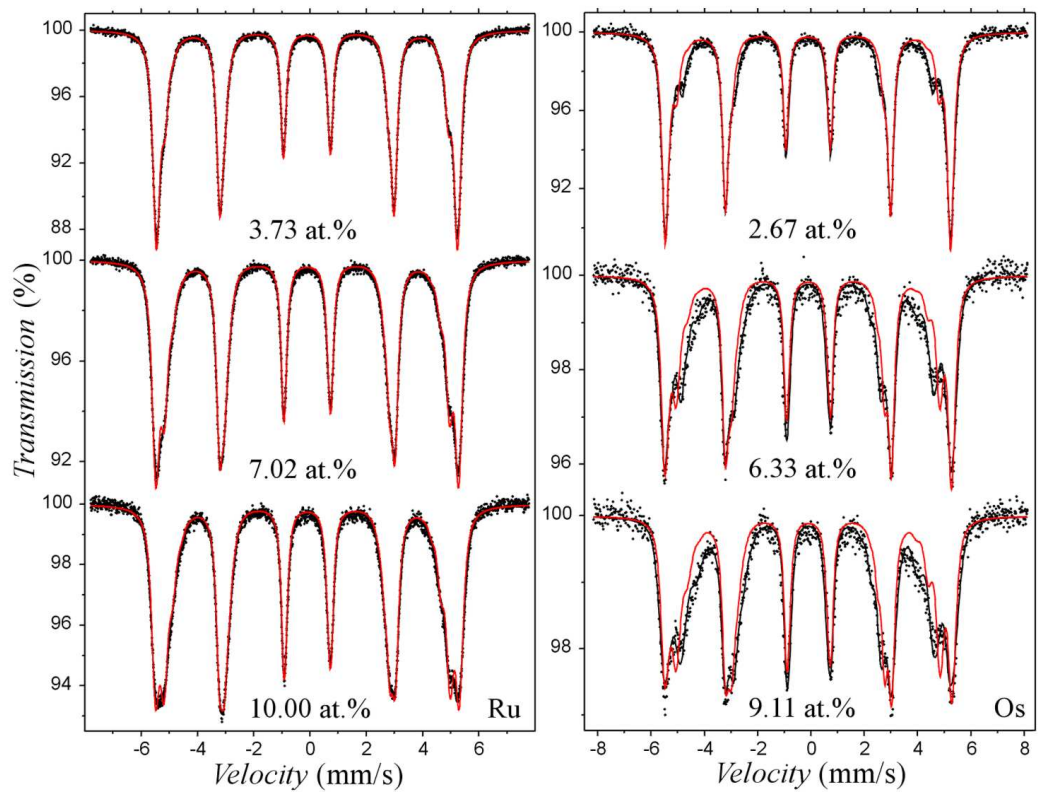

Fig. 3. Mössbauer spectra obtained for various concentrations of Ru and Os. Red lines correspond to the perturbations of the charge and spin density obtained from the $a b$ initio calculations.

Figure 2 shows selected results of the ab initio calculations. Namely, the variation of the electron density versus distance $r$ from the impurity is shown for
$\mathrm{Cr}, \mathrm{Ru}$ and Os. The electron density variation $\Delta \rho$ is taken relative to the density due to the distant impurity. The variation of the electron density is re-scaled to 
the variation of the isomer shift $\Delta S$ by using calibration constant -0.291 a.u. ${ }^{3} \mathrm{~mm} \mathrm{~s}^{-1}[31]$. Similar variation $\Delta B$ for the hyperfine field is shown as well. Results of $a b i n i$ tio calculations for all $\mathrm{d}$ impurities soluble in $\alpha$-Fe are to be published separately, soon.

Figure 3 shows selected spectra for various concentrations of $\mathrm{Ru}$ and Os in iron. Black solid line is the result of fit by Gmbernz program, while the red line was obtained by means of the similar fit, albeit with the $\Delta S_{n}$ and $\Delta B_{n}$ $(n=1,2,3)$ taken from the $a b$ initio results.

\section{Conclusions}

It is surprising that such simple phenomenological model like CAM reproduces quite reasonably the average isomer shift. Perturbations due to the impurity in the first co-ordination shell are reproduced a bit poorer, but still reasonably. The volume effect (unaccounted for in the simple version of the CAM used here) is visible as the separation of lines in Fig. 1a describing 3d, 4d and $5 \mathrm{~d}$ impurities, respectively. The volume effect could be easily taken into account, while performing ab initio calculations [32]. The positive values of the parameter $A$ are caused by the effect of sucking electrons from iron by the impurity having positive potential against iron (the calibration constant for iron is negative [31]). Negative values of the parameter $B$ are due to the same effect, i.e., to the increased electron density for impurities having higher density than iron.

$A b$ initio results show that the impurity is able to perturb electron and spin density to about third coordination shell. This result is consistent with the large body of the experimental data processed by Gmbernz. Oscillations of the charge and spin density around impurity are seen both in calculations and experiment particularly for $\mathrm{Ru}$ and Os.

Spectra fitted basing on the ab initio results depart from the experimental data with the increasing impurity concentration. This fact is understandable as $a b i n i$ tio calculations were performed for the isolated impurity within large super-cell. Currently, it is practically impossible to perform reliable calculations for the random alloy except for the very diluted limit. The number of configurations to be taken into account for the more concentrated alloy prevents detailed calculations.

\section{Acknowledgments}

Professor Krzysztof Ruebenbauer, Pedagogical University, Kraków, Poland is thanked for contribution to the data evaluation and models development. Dr. Jan Żukrowski, AGH University of Science and Technology, Kraków, Poland is thanked for alloys preparation.

\section{References}

[1] A. Błachowski, Acta Phys. Pol. A 114, 1563 (2008).

[2] A.R. Miedema, F. van der Woude, Physica 100B, 145 (1980).
[3] A.R. Miedema, Physica B 182, 1 (1992).

[4] I. Vincze, A.T. Aldred, Solid State Commun. 17, 639 (1975).

[5] S.M. Dubiel, W. Zinn, Phys. Rev. B 26, 1574 (1982).

[6] S.M. Dubiel, W. Zinn, J. Magn. Magn. Mater. 28, 261 (1982).

[7] S.M. Dubiel, Phys. Rev. B 48, 4148 (1993).

[8] J. Cieślak, S.M. Dubiel, J. Alloys Compd. 350, 17 (2003).

[9] S.M. Dubiel, W. Zinn, J. Magn. Magn. Mater. 37, 237 (1983).

[10] S.M. Dubiel, J. Żukrowski, J. Magn. Magn. Mater. 23, 214 (1981).

[11] I. Vincze, I.A. Campbell, J. Phys. F: Met. Phys. 3, 647 (1973).

[12] J. Chojcan, Hyperfine Interact. 156/157, 523 (2004).

[13] S.M. Dubiel, W. Zinn, Phys. Rev. B 28, 67 (1983).

[14] I. Vincze, A.T. Aldred, Phys. Rev. B 9, 3845 (1974).

[15] S.M. Dubiel, W. Znamirowski, Hyperfine Interact. 9, 477 (1981).

[16] S.M. Dubiel, W. Zinn, Phys. Rev. B 30, 3783 (1984).

[17] S.M. Dubiel, J. Magn. Magn. Mater. 69, 206 (1987).

[18] S.M. Dubiel, Phys. Rev. B 37, 1429 (1988).

[19] A. Błachowski et al. - unpublished.

[20] A. Błachowski, K. Ruebenbauer, J. Żukrowski, J. Przewoźnik, J. Alloys Compd. 455, 47 (2008).

[21] A. Błachowski, K. Ruebenbauer, J. Żukrowski, Phys. Status Solidi B 242, 3201 (2005).

[22] A. Błachowski, K. Ruebenbauer, J. Żukrowski, J. Przewoźnik, J. Alloys Compd. 482, 23 (2009).

[23] A. Błachowski, K. Ruebenbauer, J. Żukrowski, Phys. Rev. B 73, 104423 (2006).

[24] A. Błachowski, K. Ruebenbauer, J. Żukrowski, J. Alloys Compd. 477, 4 (2009).

[25] A. Błachowski, K. Ruebenbauer, J. Żukrowski, Phys. Scr. 70, 368 (2004).

[26] A. Błachowski, K. Ruebenbauer, J. Żukrowski, Nukleonika 49, S67 (2004).

[27] A. Błachowski, K. Ruebenbauer, J. Żukrowski, J. Alloys Compd. 464, 13 (2008).

[28] A. Błachowski, K. Ruebenbauer, J. Przewoźnik, J. Żukrowski, J. Alloys Compd. 458, 96 (2008).

[29] G. Kresse, J. Furthmüller, Computer code VASP, http://cms.mpi.univie.ac.at/vasp/; G. Kresse, J. Furthmüller, Phys. Rev. B 54, 11169 (1996).

[30] P. Blaha, K. Schwarz, G.K.H. Madsen, K. Kvasnicka, J. Luitz, Computer code WIEN2k, http://www.wien2k.at; K. Schwarz, P. Blaha, G.K.H. Madsen, Comput. Phys. Commun. 147, 71 (2002).

[31] U.D. Wdowik, K. Ruebenbauer, Phys. Rev. B 76, 155118 (2007).

[32] A. Błachowski, U.D. Wdowik, K. Ruebenbauer, J. Alloys Compd. 485, 36 (2009). 